\title{
Magnetic Stray Field Detection as Guidance for Electronic Transport Measurements in the $B-T$ Phase Diagram of $\mathrm{MnSi}$
}

\author{
M. Pohlit, S. Witt, M. Akbari, C. Krellner and J. Müller* \\ Institute of Physics, Goethe-University, Frankfurt a. M., Germany
}

\begin{abstract}
In order to advance modern information technologies, progress in both the fabrication of magnetic nanostructures and of complex materials, from which small magnetic entities - like the skyrmions present in $\mathrm{MnSi}$ emerge and in developing measurement techniques are desired. Here the sensor-based stray field detection using tailor-made micro-Hall magnetometers has proven to be a versatile tool for studying the magnetization reversal of individual magnetic nanostructures, domain wall motion in thin films, as well as the local stray field close to macroscopic samples. In this article we demonstrate that the local stray field can be used to accurately map out the $B-T$ phase diagram of $\mathrm{MnSi}$ and serve as a guidance for simultaneously-performed electronic transport measurements. The presented study also serves as a proof-of-principle experiment for future combined investigations of electronic transport and magnetization focusing on electrically-contacted magnetic nanostructures.
\end{abstract}

DOI: 10.12693/APhysPolA.133.582

PACS/topics: 07.55.Ge, 72.15.Eb, 72.80.Ga, 75.30.-m

\section{Introduction}

Advancement of modern information technology over the last decades is closely intertwined with a steady progress in the development of new magnetic materials and advances in nanostructure fabrication. More recently the change from solely charge-based electronics towards more complex and more capable devices by additionally employing the spin of the electron led to fascinating new applications within the research field of 'spintronics' [1]. While some applications like the giant magnetoresistive read heads have already entered the market of consumer electronics, the development of new spintronic devices still offers numerous promises, e.g., regarding non-volatile energy-efficient memory with fast read and write speeds [2]. Fundamental research related to storing and processing data on the one hand focuses on the use of magnetic nanostructures and dipolar-coupled arrangements of nanomagnets [3, 4], while on the other hand also the manipulation of small but stable entities within magnetic materials, like domain walls or skyrmions, are under investigation [5-7]. In this regard, especially the latter objects - found e.g. in the B20 transition metal compound $\mathrm{MnSi}$ - appear to be promising candidates as their motion can be induced via spin-transfer-torque with a low current density of around $j_{c} \approx 10^{6} \mathrm{Am}^{-2}$, which is about $10^{5}$ times less of what is typically used for domain wall movement in ferromagnets [8-10]. The skyrmion phase in $\mathrm{MnSi}$, originally named A-phase, is found in moderate external fields slightly below the paramagnetic-to-helimagnetic transition in zero field at $T_{\mathrm{C}}=28.5 \div 29.5 \mathrm{~K}[9,11]$ in a relatively small pocket of the rich $B-T$ phase-diagram schematically shown in Fig. 2(a). Upon cooling below $T_{\mathrm{C}}$ at $H_{\text {ext }}=0$ or at

\footnotetext{
* corresponding author; e-mail:

j.mueller@physik.uni-frankfurt.de
}

small external magnetic fields, the interplay between the ferromagnetic exchange and the Dzyaloshinskii-Moriyainteraction (DMI) leads to a helical magnetic ordering with a relatively long wavelength $\left(\lambda_{h} \approx 190 \AA\right)$ compared to the lattice constant $(a \approx 4.56 \AA)$ that is weakly aligned along the $\langle 111\rangle$-direction [11]. Upon increasing the external field at temperatures far below $T_{\mathrm{C}}$, the helical order gets unpinned and the propagation vector becomes aligned with the external field direction in the so-called conical phase, while the moments rotate from a perpendicular arrangement relative to the propagation direction towards a field-aligned configuration. Finally, a full alignment of the spins with the external field marks the transition from the conical to the field-polarized ferromagnetic phase were the effects of the DMI are fully suppressed. Remarkably, in a small pocket in the vicinity of the helimagnetic transition temperature $T_{\mathrm{C}}$ the so-called A-phase is formed which is characterized by a hexagonal lattice of skyrmions, reminiscent of flux tubes in a superconductor. In order to perform more complex electronic transport measurements - aiming e.g. to determine the low-frequency dynamics of charge carriers in magnetic systems [12] - related to specific magnetic phases or phase transition lines, it is essential to determine the exact position in the magnetic phase diagram. In the following we show that for this purpose electronic transport measurements can be conducted on a sample the stray field of which is detected simultaneously by a micro-Hall-magnetometer.

\section{Methods and materials}

The MnSi crystal was grown using the Bridgman method. EDX analysis shows a lack of about $2 \%$ manganese from the stoichiometric ratio due to the larger vapor pressure of manganese at high temperatures. After growth, a suitably sized sample was cut and polished down to approximately $1870 \times 350 \times 100 \mu \mathrm{m}^{3}$, electronically isolated on the bottom using GE-varnish 
(a)

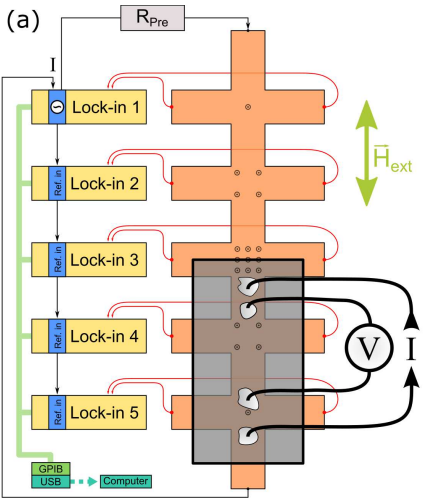

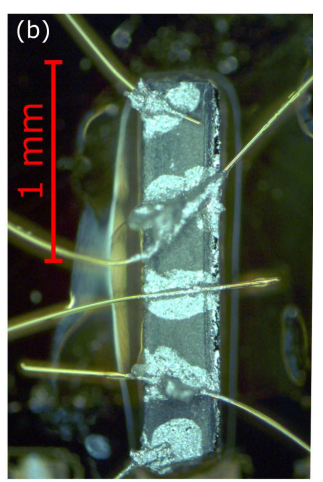

Fig. 1. (a) Simultaneous detection of a sample magnetic stray field by a micro-Hall sensor array and electronic transport measurements (schematic, sample and sensor are not drawn to scale). (b) MnSi sample contacted with gold wires glued with silver paste attached to the Hall sensor surface. Note the elongated sample shape, which minimizes demagnetization effects.

and fixed to the sensor surface, i.e. the gold top gate of a micro-Hall-magnetometer, see Fig. 1(a) and (b). The homebuilt Hall-sensor-array which provides direct access to the stray field of the MnSi sample was fabricated from a GaAs/AlGaAs 2DEG heterostructure using UV-lithography and wet chemical etching methods. The electronic read out of the adjacent Hall crosses was performed at low temperatures in a cryogenic setup using standard lock-in techniques to measure the Hall-Voltage $V_{H}$, which is induced by the perpendicular component of the sample's stray field. In a first approximation $V_{H}$ is given by the average perpendicular stray field in the active area of the Hall-cross $\left\langle B_{z}\right\rangle$, in our case $10 \times 10 \mu \mathrm{m}^{2}$, $R_{H}=V_{H} / I=1 / n e\left\langle B_{z}\right\rangle$, where $I$ denotes the applied sensor current while $n \approx 2.5 \cdot 10^{11} \mathrm{~cm}^{-2}$ is the carrier concentration at low temperatures. The detected signal is directly connected to the magnetization of the sample [13] and can be used to identify the different magnetic phases as discussed below. An external magnetic field applied parallel to the sensor plane is used to manipulate the sample magnetization.

\section{Results and discussion}

Using the set-up illustrated in Fig. 1(a), temperatureand magnetic field-dependent measurements were performed, first to demonstrate that the stray field of the sample allows for a precise mapping of the $B-T$ phase diagram of $\mathrm{MnSi}$, and second that this information can also be acquired simultaneously by electronic transport measurements. In line with standard magnetization measurements, the field-dependent acquisition of the sample stray field at several fixed temperatures allowed for an accurate determination of the location of the skyrmion phase. For this purpose the derivative of the measured Hall resistance, $\mathrm{d} R_{\mathrm{H}} / \mathrm{d} B$, in analogy to the susceptibility $\mu_{0} \mathrm{~d} M / \mathrm{d} B$ used in [14], was employed, which displays a characteristic curve shape from which the different magnetic phases are easily identified, see Fig. 2(d). A contour plot of the smoothed derivatives of a set of field sweeps shown in Fig. 2(c) is in excellent agreement with the expected $B-T$ phase diagram, $c f$. Fig. 2(a) and [14]. That the magnetic stray field and the four-wire resistance of the electrically contacted sample - exemplary for more complex electronic transport properties alike can be measured simultaneously, is illustrated for a single cooldown measurement at $\mu_{0} H_{\text {ext }} \approx 200 \mathrm{mT}$ in Fig. 2(b). Here a small dip in the stray field accompanies entering the skyrmion phase while the simultaneously measured resistance displays a more continuous reduction which is in good agreement with earlier PPMS measurements and literature results, e.g., the shape of the resistivity curve measured at $\mu_{0} H_{\text {ext }}=194 \mathrm{mT}$ in Fig. 4 of [15].

These findings demonstrate the useful combination of electrically-contacted samples and micro-Hall magnetometry, originally used to study superconducting materials [16], towards studies on complex magnetic materials like MnSi. Micro-Hall magnetometry already has proven to be a versatile tool allowing for high-resolution studies of the magnetization reversal of isolated nanostructures [17-19]. Therefore, in addition to the detection of local magnetic entities, as e.g. the motion of domain walls [20,21], numerous potential applications in the field of 'spintronics' and skyrmion physics (e.g. for studying their dynamical transport and magnetic properties) are conceivable. Also, since the combination of micro-Hall magnetometry with direct-write magnetic nanostructures using focused electron beam induced deposition has proven to be extremely fruitful in the past [17-19], simultaneous magnetic and transport studies of $2 \mathrm{D}$ and $3 \mathrm{D}$ nanostructures become possible.

\section{Acknowledgments}

The wafer material used in the sensor fabrication was kindly provided by Dr. Jürgen Weis (MPI for Solid State Research, Stuttgart, Germany).

\section{References}

[1] S.A. Wolf, D.D. Awschalom, R.A. Buhrman, J.M. Daughton, S. von Molnár, M.L. Roukes, A.Y. Chtchelkanova, D. M. Treger, Science 294, 1488 (2001).

[2] J. Åkerman, Science 308, 508 (2005).

[3] R.P. Cowburn, M.E. Welland, Science 287, 1466 (2000).

[4] A. Imre, G. Csaba, L. Ji, A. Orlov, G.H. Bernstein, W. Porod, Science 311, 205 (2006).

[5] S.S.P. Parkin, M. Hayashi, L. Thomas, Science $\mathbf{3 2 0}$ 190 (2008).

[6] D.A. Allwood, G. Xiong, C.C. Faulkner, D. Atkinson, D. Petit, R.P. Cowburn, Science 309, 1688 (2005).

[7] X. Zhang, M. Ezawa, Y. Zhou, Sci. Rep. 5, 9400 (2015).

[8] F. Jonietz, S. Mühlbauer, C. Pfleiderer, A. Neubauer, W. Münzer, A. Bauer, T. Adams, R. Georgii, P. Böni, R.A. Duine, K. Everschor, M. Garst, A. Rosch, Science 330, 1648 (2010). 
[9] T. Schulz, R. Ritz, A. Bauer, M. Halder, M. Wagner, C. Franz, C. Pfleiderer, K. Everschor, M. Garst, A. Rosch, Nature Phys. 8, 301 (2012).

[10] M. Tsoi, R.E. Fontana, S.S.P. Parkin, Appl. Phys. Lett. 83, 2617 (2003).

[11] S. Mühlbauer, B. Binz, F. Jonietz, C. Pfleiderer, A. Rosch, A. Neubauer, R. Georgii, P. Böni, Science 323, 915 (2009).

[12] P. Das, A. Amyan, J. Brandenburg, J. Müller, P. Xiong, S. von Molnár, Z. Fisk, Phys. Rev. B $\mathbf{8 6}$ 184425 (2012).

[13] M. Pohlit, I. Stockem, F. Porrati, M. Huth, C. Schröder, J. Müller, J. Appl. Phys. 120, 142103 (2016).

[14] A. Bauer, C. Pfleiderer, Phys. Rev. B 85, 214418 (2012).

[15] I.I. Lobanova, V.V. Glushkov, N.E. Sluchanko, S.V. Demishev, Sci. Rep. 6, 22101 (2016).
[16] D.T. Fuchs, E. Zeldov, D. Majer, R.A. Doyle, T. Tamegai, S. Ooi, M. Konczykowski, Phys. Rev. B 54, R796 (1996).

[17] M. Pohlit, F. Porrati, M. Huth, Y. Ohno, H. Ohno, J. Müller, J. Appl. Phys. 117, 17C746 (2015).

[18] F. Porrati, M. Pohlit, J. Müller, S. Barth, F. Biegger, C. Gspan, H. Plank, M. Huth, Nanotech. 26, 475701 (2015).

[19] M. Pohlit, F. Porrati, M. Huth, Y. Ohno, H. Ohno, J. Müller, J. Magn. Magn. Mat. 400, 206 (2016).

[20] K.S. Novoselov, A.K. Geim, S.V. Dubonos, E.W. Hill, I.V. Grigorieva, Nature 426, 812 (2003).

[21] P. Das, F. Porrati, S. Wirth, A. Bajpai, M. Huth, Y. Ohno, H. Ohno, J. Müller, Appl. Phys. Lett. 97, 042507 (2010). (a)

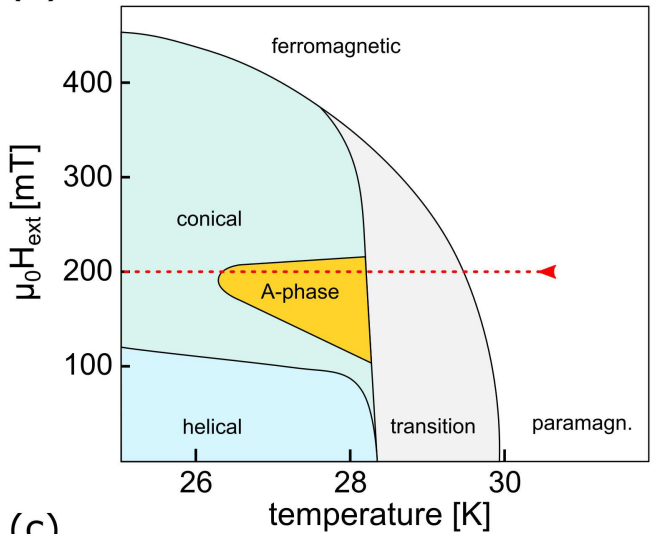

(c)

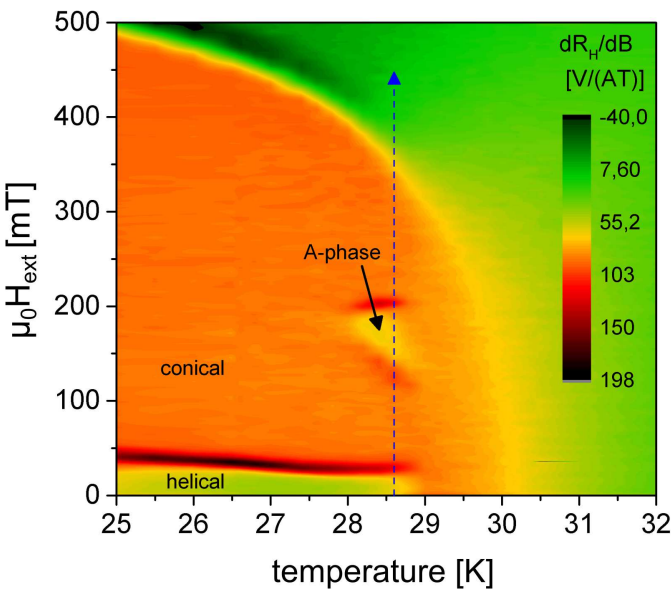

(b)
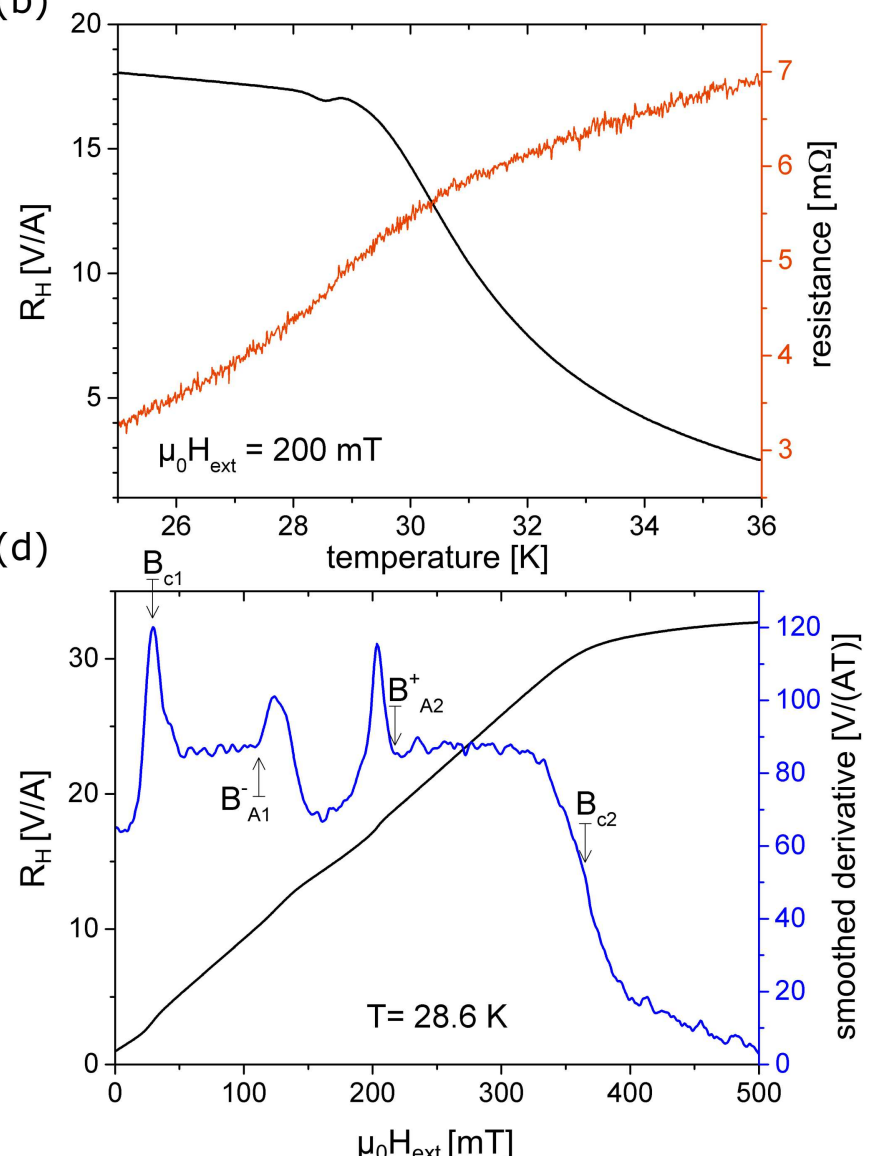

Fig. 2. (a) Phase diagram of MnSi [14]. Dashed red line illustrates a single cooldown measurement at a fixed external magnetic field as presented in (b). (b) The sensor Hall resistance $R_{\mathrm{H}}$, representing the magnetization of the sample, displays a small minimum around the upper skyrmion phase boundary during cooldown at $\mu_{0} H_{\text {ext }}=200 \mathrm{mT}$ (black line). The simultaneously-acquired four-point-resistance of the sample displays the typical decrease around the phase transition (red curve). ( $I_{\text {sensor }} \approx 5 \mu \mathrm{A}$ at $f=117 \mathrm{~Hz}, I_{\mathrm{MnSi}} \approx 500 \mu \mathrm{A}$ at $f=11 \mathrm{~Hz}$.) (c) Experimental phase diagram deduced from field-dependent stray field measurements at various fixed temperatures (field sweeps between $\mp 0.5 \mathrm{~T}$ sweep rate $15 \mathrm{mT} / \mathrm{min})$. Depicted is the smoothed derivative $\mathrm{d} R_{\mathrm{H}} / \mathrm{d} B$ of the Hall resistance in positive external fields. The dashed blue line illustrates a representative measurement taken at $T=28.6 \mathrm{~K}$ as depicted in $(\mathrm{d})$. (d) Hall resistance of a single field sweep across the A-phase and the corresponding derivative $\mathrm{d} R_{\mathrm{H}} / \mathrm{d} B$ with characteristic extrema. $B_{\mathrm{c} 1}$ marks the transition from the helical into the conical phase, $B_{\mathrm{A} 1}^{-}$represents the transition entering the skyrmion phase, while $B_{\mathrm{A} 2}^{+}$marks the upper phase boundary (designations in accordance with [14]). At $B_{\mathrm{c} 2}$ the system enters the field-polarized ferromagnetic state. Note that the absolute temperature values where the different phases are observed appear to be about $1 \mathrm{~K}$ larger than the temperatures reported in [14]. This effect might be attributed to a temperature gradient between the sample and the thermometer. 